\title{
Congenital tethered spinal cord syndrome in adults
}

\author{
Bermans J. Iskandar, M.D., Benjamin B. Fulmer, M.D., Mark N. Hadley, M.D., \\ AND W. JERRY OAKES, M.D.
}

Department of Neurological Surgery, University of Wisconsin Hospital and Clinics, Madison

Wisconsin; Division of Pediatric Neurosurgery, Children's Hospital, Birmingham; and Department of Neurosurgery, University of Alabama at Birmingham, Birmingham, Alabama

\begin{abstract}
Object. The management of tethered cord syndrome with onset of symptomatology occurring in adulthood remains controversial, although the necessity of early surgery in the pediatric tethered cord syndrome population is well established. To ascertain the results of surgery in adult patients with this anomaly, the authors undertook a retrospective review of 34 cases.

Methods. The authors studied the hospital records of 34 consecutive patients who presented in adulthood with tethered cord syndrome and conducted follow-up phone interviews with 28 of them. The population consisted of 12 men and 22 women, ranging in age from 18 to 70 years (mean 34 years). The most common presenting feature was pain, followed by weakness and incontinence. All patients underwent surgery. The most common operative findings were tight filum terminale, split cord malformation, and lipomyelomeningocele, paralleling those observed in pediatric studies.

Long-term surgical results and patient outcome ratings were encouraging. After a mean clinical follow-up period of 4 years, significant improvement occurred in 22 of 27 patients presenting with pain, 13 of 27 patients with motor or sensory dysfunction, and 11 of 18 patients with bowel and bladder disturbance. In addition, telephone interviews were obtained after a period of 8.6 years. Twenty-two $(79 \%)$ of 28 patients called the operation a long-term success; 21 (75\%) of 28 patients believed that they had significant postoperative improvement (and not just stabilization) in pain and/or neurological function. Surgical complications were generally minor.

Surgery-related complications included one cerebrospinal fluid leak and five pseudomeningoceles. Two patients complained of worsening motor or sensory dysfunction and one of worsening bladder dysfunction, whereas four patients experienced persistent pain and one complained of worsening pain postoperatively. According to the patients, the vast majority of these complications were minor.

Nineteen (86\%) of 22 employed patients returned to work after surgery. Two (33\%) of six patients who were not employed before surgery worked full time postoperatively. Only two of the 28 patients interviewed had received Workers' Compensation benefits; both of these had good outcomes and returned to work.

Conclusions. Tethered spinal cord syndrome in adults is an uncommon entity that can become symptomatic.

Although surgery in adults involves greater risk of neurological injury than in children, it is a low-risk procedure with encouraging results. Because neurological deficits are generally irreversible, early surgery is recommended.
\end{abstract}

\section{KEY WORDS • tethered spinal cord • spina bifida • occult spinal dysraphism • congenital anomaly $\bullet$ adult}

Occult spinal dysraphism represents a spectrum of congenital anomalies that can cause rostrocaudal traction on the spinal cord. This "TCS" occurs commonly in children, causing pain, neurological deficits, and bowel and bladder dysfunction. ${ }^{1,3,9}$ It is well established in the literature that surgical spinal cord untethering in children is likely to prevent the development of new neurological deficits, as well as the progression of old ones. . $^{1,3,4,13}$ Patients with OSD may reach adulthood with their disease undiagnosed, despite obvious cutaneous anomalies or orthopedic deformities. The development of new symptoms (especially pain) or the progression of previously inconspicuous established deficits brings these patients to medical attention.

Abbreviations used in this paper: OSD = occult spinal dysraphism; TCS $=$ tethered cord syndrome.
Other authors have carefully described the clinical and radiographic features of adult patients who presented with congenital TCS, as well as the results of surgery in this group. ., $, 8,10-13,15-17$ The aim of this paper is to describe the results of surgery performed in a group of adult patients with OSD, with emphasis on long-term outcomes, work history, and the issue of Workers' Compensation.

\section{CLINICAL MATERIAL AND METHODS}

We retrospectively reviewed the medical records, as well as the radiographic and operative reports of 34 consecutive adult patients who presented with congenital TCS between 1971 and 1995. All of the patients underwent surgical untethering of the spinal cord as previously described in the literature ${ }^{9}$ none of these was a repeated surgery. Because the study population consisted of con- 


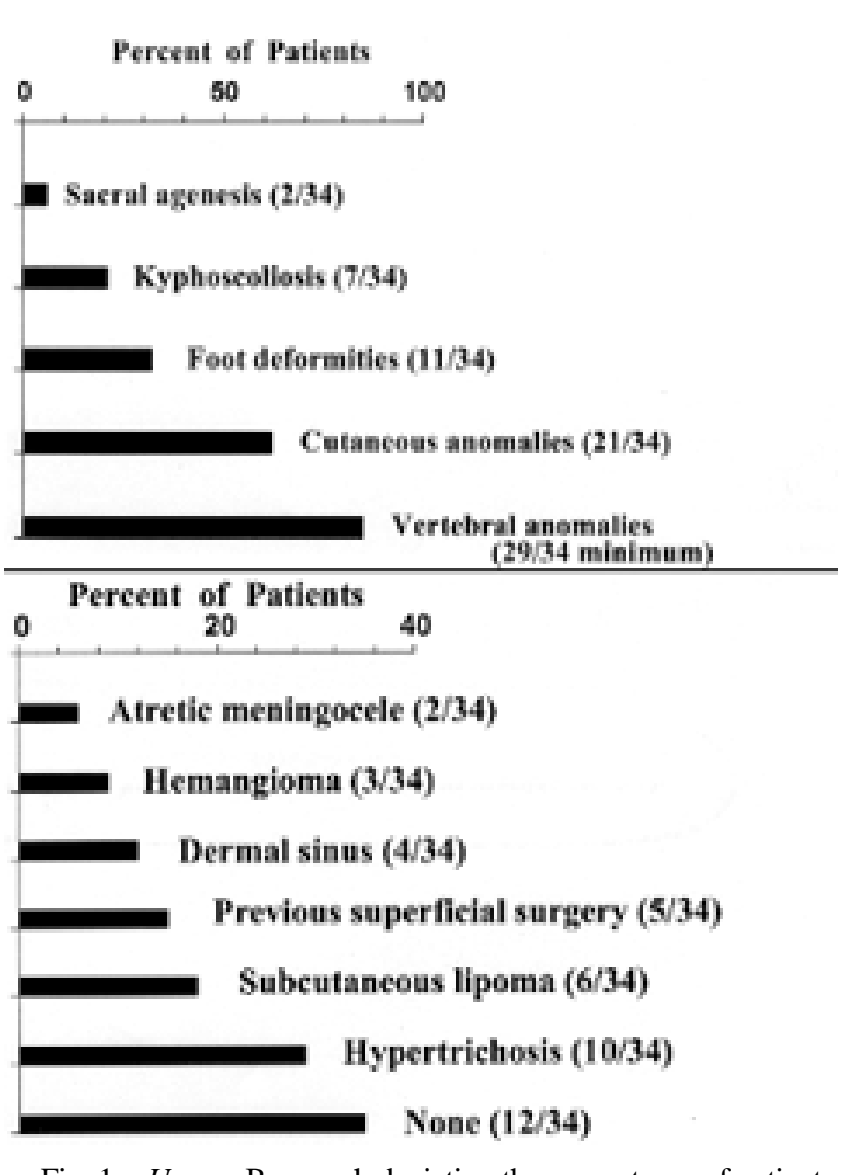

Fig. 1. Upper: Bar graph depicting the percentages of patients with associated anomalies. Lower: Bar graph showing the percentages of patients with cutaneous anomalies. The term "previous superficial surgery" is used to describe a number of patients who underwent previous operations for cutaneous deformities. These earlier operations did not include an intraspinal exploration.

genital, not acquired, spinal cord tethering, no patients with postmyelomeningocele tethered cord were included in this study. One patient who had presented at the age of 70 years was not considered to be a surgical candidate and was excluded.

The data were analyzed for type of presentation and indications for surgery. Surgical results were collected from the patients' discharge summaries as well as subsequent clinic notes; the follow-up period ranged from 1 week to 17 years, with a mean of 4 years and a median of 2 years. In addition, long-term follow-up interviews were obtained by telephone in 28 of the 34 patients, in which a standard set of questions was asked. The follow-up time period for these interviews ranged from 1 to 25 years, with a mean of 8.6 and a median of 8.5 years.

\section{RESULTS}

There were 12 men and 22 women in the group. The patients' ages ranged from 18 to 70 years; the median and mean ages at surgery were both 34 years. Although the majority of patients had the cutaneous stigmata of dysraphism (21 patients with cutaneous anomalies and two

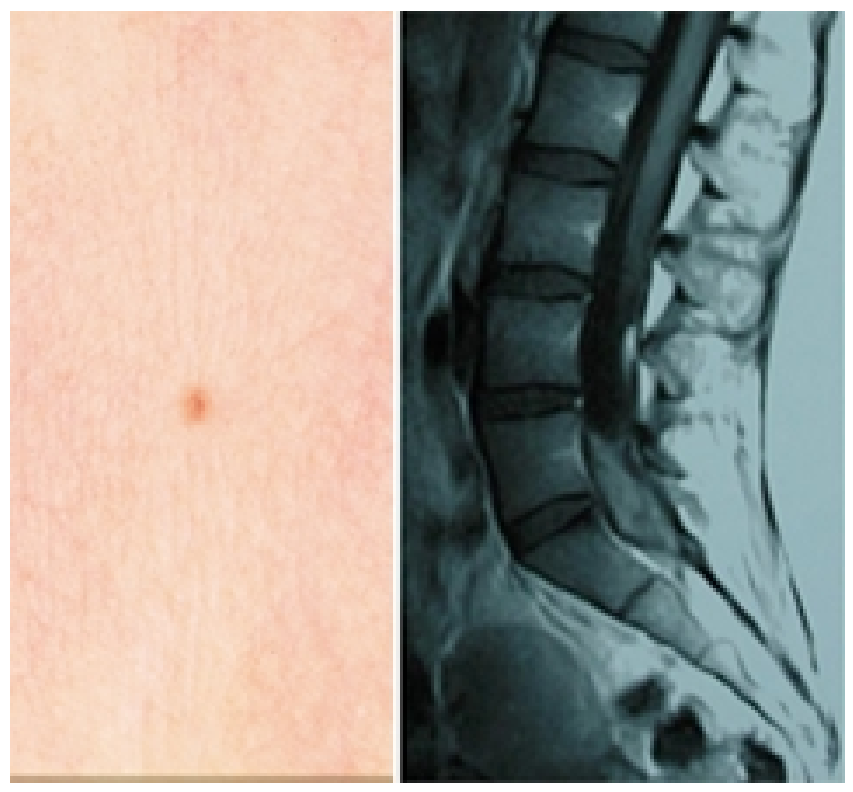

Fig. 2. Left: Intraoperative photograph showing a flat faint skin hemangioma and dermal sinus opening, two typical stigmata of OSD. Right: Magnetic resonance image revealing a conus medullaris at L-3, a thick and fatty filum terminale, and evidence of a mass or fluid of the sacral spinal canal.

patients with sacral agenesis or hypogenesis), these were either disregarded or had been treated surgically in the past without attention to the underlying spinal cord malformation (Figs. 1 and 2). Instead, the presenting complaints included pain in 27 patients, motor or sensory symptoms or signs in 27 , and bowel and bladder dysfunction in 18. Vertebral anomalies were present in 30 patients. Although strongly suspected, no documentation of the presence or absence of such bone anomalies was found in the records of the other four patients.

Intraoperative findings (Figs. 3 and 4) included a thick and/or fatty filum terminale in 19 patients, a spinal cord lipoma or lipomyelomeningocele in 15, a split cord malformation in 13, meningocele manqué in eight, terminal

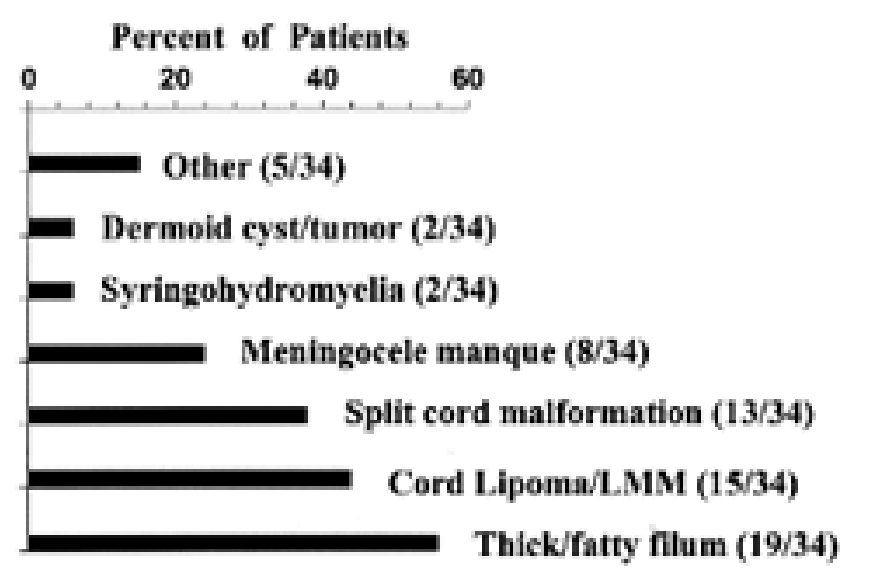

Fig. 3. Bar graph displaying the percentages of patients with the various aspects of OSD. $\mathrm{LMM}=$ lipomyelo-meningocele. 


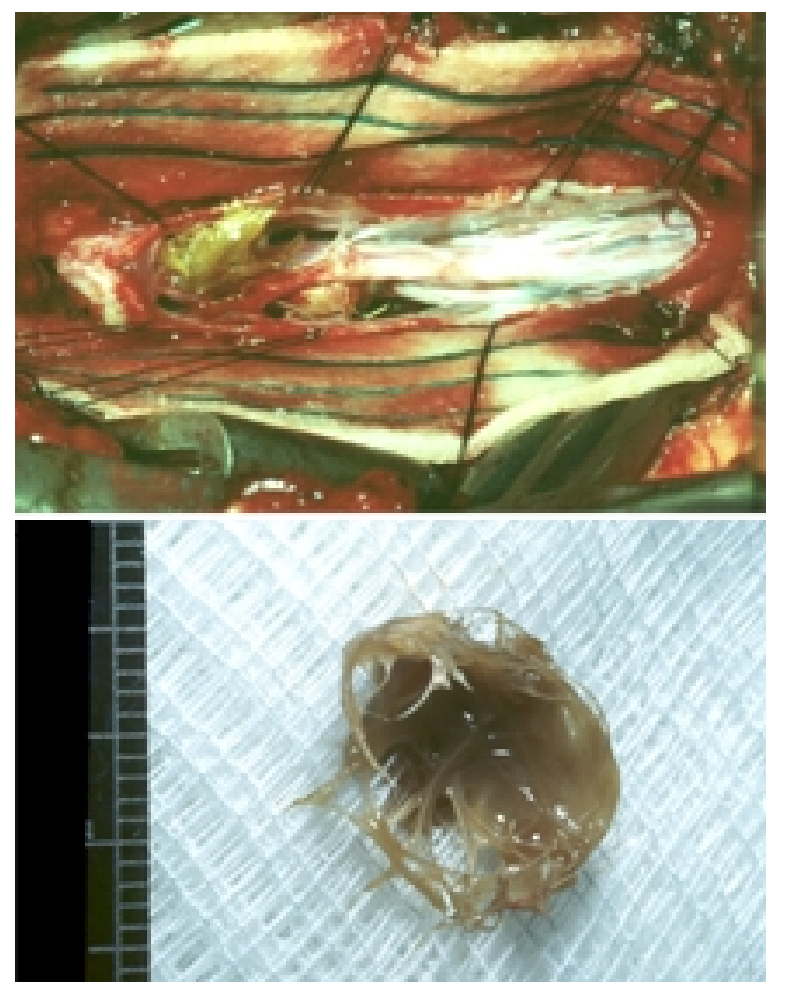

Fig. 4. Upper: Intraoperatively it was noted that the sinus tract led to a cauda equina dermoid that was severely calcified and adherent to the nerve roots. Lower: Photograph of a "hair ball" that accompanied the dermoid.

syringohydromyelia in two, a dermoid cyst in two, and ventral bands, a neurenteric cyst, an atretic meningocele, an epi-thelialized myelomeningocele, and an arachnoid cyst in one patient each. Some of these lesions were diagnosed preoperatively by using myelograms and postmyelogram computerized tomography scans and/or magnetic resonance images.

Surgical results that had been recorded by the physician in the clinic notes were collected for all 34 patients (Fig. 5). Of the 27 patients with pain, 22 improved, one worsened, and four were unchanged. Of the 27 patients with motor or sensory complaints, 13 improved, two worsened, and 12 were unchanged. Of the 18 patients with bowel and bladder dysfunction, 11 improved, one worsened, and six were unchanged. Deteriorations were generally mild and did not affect the patient's functional level.

Long-term results recorded during telephone interviews were collected for 28 of the 34 patients. When asked if the operation was a success, 22 patients responded yes and six said no. When asked if there was improvement in the presenting symptomatology, 21 responded in the affirmative. Thirteen of the patients described complete resolution of their symptoms; five patients stated that, although some symptoms had improved or resolved, others remained unchanged; another four patients reported improvement in some symptoms and deterioration in others; two patients said that their symptoms had worsened postoperatively; and four others detected no change. According to the majority of patients who reported postoperative symptomatic

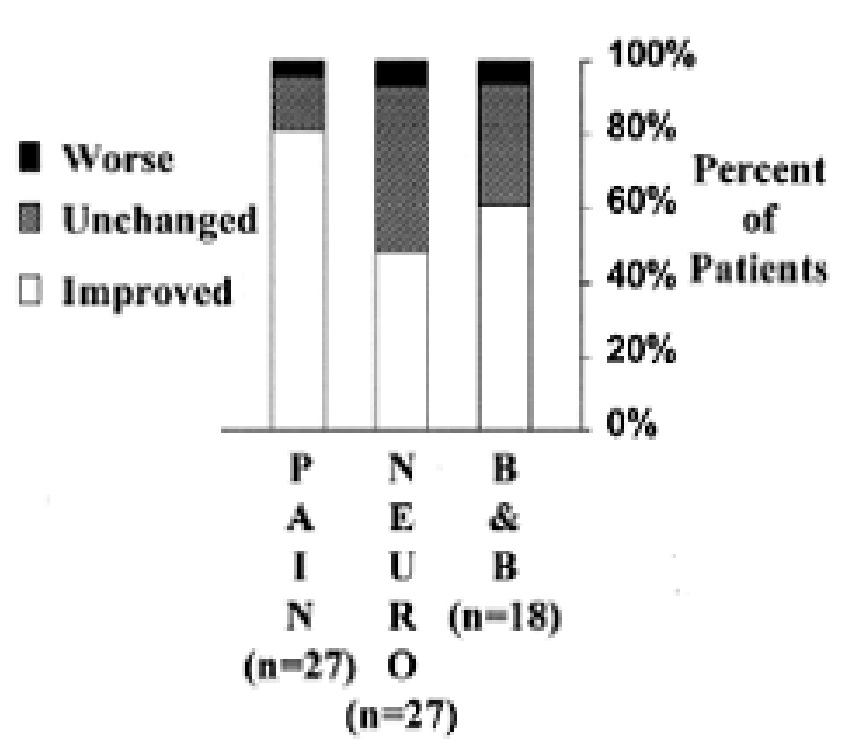

Fig. 5. Bar graph showing the results of surgery on different presenting symptoms and signs: pain, neurological problems (NEURO), and bowel and bladder dysfunction (B \& B). The mean follow-up duration was 4 years.

deteriorations, these were of minor functional significance. One particular patient with terminal syringohydromyelia as well as other spinal defects underwent several surgical procedures on the syrinx. Improvement or resolution of the symptoms and signs, as well as radiographic improvement in the size of the syrinx, lasted several months to 2 years after each operation.

Long-term work history was also established by telephone interview. Of 22 patients who had worked preoperatively, 19 returned to their jobs after surgery. The other three patients stopped working within months of surgery due to circumstances unrelated to the surgery. One underwent a coronary artery bypass operation shortly after his spinal operation; another obtained the disability status that he had decided to seek preoperatively; and the third retired from her job in anesthesiology, a decision that she had also made preoperatively. Of six patients who did not work preoperatively, two had full-time jobs after surgery. One of these patients had just turned 18 years of age; therefore, the surgery may have been inconsequential to her obtaining a job postoperatively. When asked, only two of the 28 patients stated that they had sought Workers' Compensation benefits preoperatively. Both of these patients returned to work after surgery.

Nonneurological surgical complications consisted of one spinal fluid leak and five pseudomeningoceles, all of which were successfully treated either conservatively or surgically.

\section{DISCUSSION}

It is commonly believed that children who have a congenital tethered cord benefit from surgical untethering because it prevents neurological deterioration., ${ }^{3,9,14}$ However, controversy has surrounded the issue of surgery in 
newly diagnosed adult patients. The incidence of OSD is unknown and, although it is likely that some patients remain asymptomatic and a diagnosis is never made, a subset of patients with the congenital syndrome develop progressive symptoms and signs in adulthood. ${ }^{2,5,10,12,13}$ Such deterioration may occur after sudden movements that cause traction on the spinal cord. ${ }^{10}$ These may include bending movements, the lithotomy position during childbirth, movement occurring during motor vehicle accidents, and others. Would surgery in adulthood cause similar long-term improvement in function and, if so, at what cost of complications?

In this paper we describe our experience with 34 adult patients who underwent surgical untethering operations. The clinical presentation of these patients is similar to that reported in previous studies, $2,5,6,8,10,11,13,16,17$ stressing the predominance of patients presenting with pain-this being the major difference between adult and pediatric presentations. The surgical complication rate is low. However, it is slightly higher than that in children with the same disease if one were to compare the data with those of pediatric studies. ${ }^{1,3,4,9}$ Long-term results are encouraging, indicating the efficacy of surgery in at least some of these patients. In addition, it is obvious that in a substantial number of our patients, the benefits of surgery were not limited to the prevention of deficits; instead we can infer from our data that newly acquired neurological dysfunction in adulthood is often reversible with surgery.

The diagnosis of OSD in an asymptomatic adult patient is still controversial. Adults are thought to have completed their growth curve; therefore, they are less likely to show any neurological deterioration because there has already been rapid spinal column growth in the setting of a spinal cord that is tethered caudally. However, the issue of mechanical stretching of the spine due to trauma or specific postures plays an important role in the development of new symptoms in these patients. In fact, a significant number of our patients, as well as patients reported in other studies, have been shown to have such a history. ${ }^{10,18}$ It is impossible to tell how many patients actually remain asymptomatic because, presumably, these persons will rarely seek medical attention. All patients with this condition whom we have evaluated have demonstrated some deterioration in function related to the spinal anomaly. It is difficult to know if asymptomatic patients are not being referred to appropriate centers or if they just do not exist. Often, patients may be unaware of their existing neurological deficits or may underestimate or minimize their significance. A striking example of this is a 48-year-old woman with a lipomyelomeningocele who developed saddle anesthesia and frequent urinary tract infections after being in the lithotomy position during childbirth. (This patient is not included in this study because she was evaluated only recently and has still not undergone surgical untethering.) Her doctor attributed the saddle numbness to an episiotomy incision, even though the numbness extended to the knee joints posteriorly. She did not attribute the urinary tract infections to her lifelong back problem. This same patient also had "hammer toes" all of her life, for which she underwent surgical correction. This case underlines the importance of a detailed neurological his- tory and examination in this group of patients because, initially, this woman described herself as "asymptomatic."

The importance of this article is that it is the only report with long-term surgical outcome data in an adult population with TCS. Although telephone interviews may introduce significant bias, they do supply some information regarding the satisfaction of the patients with their postoperative conditions. In a more objective fashion, we did ascertain how the operation affected the patients' abilities to work and be productive. This information becomes especially important when one considers the number of patients with back pain due to degenerative disease who seek Workers' Compensation benefits and may not show long-term benefit from surgical procedures. We have demonstrated that our patients are distinctly different from these patients; rather, they are similar to the pediatric population with tethered cord in that they are likely to benefit from surgery and become (or continue to be) part of the productive work force.

Finally, the type of surgical untethering needed in adult patients with OSD is beyond the scope of this article., ${ }^{4,9}$ Interested readers are referred to representative articles in the literature that offer discussions of these techniques. It is important to state, however, that in our experience, tethered cord operations in adults, in general, have been more difficult to perform than similar procedures in children. In adult patients, intradural structures are frequently scarred and surrounded by significant arachnoidal adhesions that are very likely to contribute to the patients' symptoms; this scarring is often severe enough to make it more difficult to dissect the edge of the spinal cord from the surrounding dura. In addition, the anatomy may be very unusual and confusing. Therefore, caution should be exercised during the dissection, and preoperative computerized tomography and magnetic resonance images must be studied very carefully before surgery.

Since the original publication of these data, the authors have surgically treated, independently, numerous other adult patients with congenital tethering of the spinal cord, and they have achieved similar successes. The use of intraoperative electrophysiological monitoring continues to be controversial, even among the different authors of this paper. In the recent experience of one of the authors (B.J.I.), for instance, intraoperative electromyography has been useful in differentiating between scar tissue and neural structures. It remains true, however, that the most important factor in preventing postoperative neurological deterioration is not any particular technique of surgery and monitoring but the experience of the surgeon in handling these complex congenital anomalies.

\section{Acknowledgments}

The authors thank Timothy George, Dawn Chestnut, and Faye Whitt for their kind assistance in providing patient information and records.

\section{References}

1. Anderson FM: Occult spinal dysraphism: a series of 73 cases. Pediatrics 55:826-835, 1975

2. Balagura S: Late neurological dysfunction in adult lumbosacral lipoma with tethered cord. Neurosurgery 15:724-726, 1984 


\section{Congenital tethered cord syndrome in adults}

3. Hoffman HJ, Hendrick EB, Humphreys RP: The tethered spinal cord: its protean manifestations, diagnosis and surgical correction. Childs Brain 2:145-155, 1976

4. James CCM, Lassman LP: Spina Bifida Occulta: Orthopedic, Radiological and Neurosurgical Aspects. London: Academic Press, 1981

5. Klekamp J, Raimondi AJ, Samii M: Occult dysraphism in adulthood: clinical course and management. Childs Nerv Syst 10: 312-320, 1994

6. Kondo A, Kato K, Kanai S, et al: Bladder dysfunction secondary to tethered cord syndrome in adults: is it curable? J Urol 135:313-316, 1986

7. Lonton AP, O'Sullivan AM, Loughlin AM: Spina bifida adults. Z Kinderchir 38 (Suppl 2):110-112, 1983

8. Maroun FB, Jacob JC: Adult diastematomyelia. Can J Neurol Sci 21:365, 1994 (Letter)

9. Oakes WJ: Tethered spinal cord, intramedullary spinal lipoma, and lipomyelomeningocele, in Rengachary SS, Wilkins RH (eds): Neurosurgical Operative Atlas. Park Ridge, IL: American Association of Neurological Surgeons, 1992, pp 133-141

10. Pang D, Wilberger JE Jr: Tethered cord syndrome in adults. J Neurosurg 57:32-47, 1982

11. Prasad VS, Sengar RL, Sahu BP, et al: Diastematomyelia in adults. Modern imaging and operative treatment. Clin Imaging 19:270-274, 1995

12. Russell NA, Benoit BG, Joaquin AJ: Diastematomyelia in adults. A review. Pediatr Neurosurg 16:252-257, 1990/91
13. Salvati M, Orlando Ramundo E, Artico M, et al: The tethered cord syndrome in the adult. Report of three cases and review of the literature. Zentralbl Neurochir 51:91-93, 1990

14. Satar N, Bauer SB, Shefner J, et al: The effects of delayed diagnosis and treatments in patients with an occult spinal dysraphism. J Urol 154:754-758, 1995

15. Simon RH, Donaldson JO, Ramsby GR: Tethered spinal cord in adult siblings. Neurosurgery 8:241-244, 1981

16. Sperke N, Spring A, Weis G, et al: Das Tethered-cord-Syndrom beim Erwachsenen als Ursache für Blasenfunktionsstörungen. Urologe 29:345-347, 1990

17. Watteau JP, Dumont O, Kaeckenbeeck B, et al: Syndrome de la corde étirée avec troubles urinaires chez l'adulte. Faut-il operer? Acta Urol Belg 58:133-136, 1990

18. Yamada S, Iacono RP, Andrade T, et al: Pathophysiology of tethered cord syndrome. Neurosurg Clin North Am 6: 311-323, 1995

Manuscript received July 17, 1997.

Accepted in final form December 19, 1997.

An earlier version of this paper was published in $\mathbf{J}$ Neurosurg 88:958-961, 1998.

Address reprint requests to: Bermans J. Iskandar, M.D., Department of Neurological Surgery, University of Wisconsin Hospita and Clinics, 600 Highland Avenue, K4/832, Madison, Wisconsin 53792. 